\title{
LAS TAREAS VIVENCIALES Y SUS IMPLICACIONES EN EL DESARROLLO DE LA COMPETENCIA DISCURSIVA EN EL APRENDIZAJE DEL INGLÉS COMO LENGUA EXTRANJERA
}

\section{VIVENTIAL TASKS AND ITS IMPLICATIONS FOR THE DEVELOPMENT OF DISCOURSE COMPETENCE IN LEARNING ENGLISH AS A FOREIGN LANGUAGE}

\author{
Juan De Dios Marín Murillo \\ Pablo Andrés Aristizabal Uribe ${ }^{2}$ \\ Juan Carlos González Sánchez ${ }^{3}$
}

\footnotetext{
1 Código Orcid: orcid.org/0000-0001-6541-1204, centro.idiomas@usbmed.edu.co, 5145600, Universidad de San Buenaventura, Medellín, Colombia. Coordinador de idiomas. Candidato a Magister en Educación.

2 Código Orcid: orcid.org/0000-0002-6725-2072, pablo.aristizabal@usbmed.edu.co, 5145600, Universidad de San Buenaventura, Medellín, Colombia. Docente de inglés. Candidato a Magister en Educación.

3 Código Orcid: https://orcid.org/0000-0002-44124334, jcgonzalez@uco.edu.co, Universidad Católica de Oriente. Docente Asociado. Facultad de Ciencias de la Educación. Magister en Educación.
}

\section{RESUMEN}

Este artículo presenta los resultados de un proyecto de investigación-acción enfocado en verificar el nivel de apropiación de la competencia discursiva entre los estudiantes. Este estudio se llevó a cabo en la Universidad de San Buenaventura Medellín desde el nivel 9 al 10 de inglés utilizando tareas vivenciales con ocho estudiantes. Todas las tareas se guiaron con 
un objetivo comunicativo integrando las cuatro habilidades (hablar, escuchar, escribir y leer; enfoque del lenguaje integrado) para mejorar la apropiación de la competencia discursiva de los participantes teniendo en cuenta el programa de estudios y las necesidades e intereses de los ellos. Los resultados muestran las mejoras de los alumnos en relación a su competencia discursiva, tanto oral como escrita. Además, los hallazgos evidencian que la implementación de tareas vivenciales fue significativa por factores relacionados con el enfoque del lenguaje integrado, enseñanza del lenguaje basado en tareas y los objetivos comunicativos con respecto al programa de estudios y las necesidades e intereses del estudiante.

PALABRAS CLAVE: tarea, confianza, discurso, apropiación.

\section{ABSTRACT}

This article presents the results of an actionresearch project focuses on verifying the level of appropriation of the discursive competence among the students. This study was carried out at the University of San Buenaventura Medellín from level 9 to 10 of English program using vivential tasks with eight students. All tasks were guided with a communicative goal integrating the four skills (speaking, listening, writing and reading; whole language approach) in order to improve learners' appropriation of discursive competence taking into account the syllabus and student's needs and interests. The results show learners' improvements in their discursive competence such as oral as written. In addition, findings evidence that implementation of vivential tasks was meaningful by factors related to Whole language approach, Task-based language teaching and Communicative goals regards to the syllabus and learner's needs and interests.

KEYWORDS: task, confidence, speech, appropriation.

\section{INTRODUCCIÓN}

El aprendizaje del idioma inglés, sus métodos y formas de enseñanza cobran cada vez más interés para las diversas ciencias de la educación y para otras ciencias humanas. Su importancia estriba en que aprender inglés es altamente relevante en el contexto de un mundo globalizado (Flores, 2016). Si bien, el inglés es considerado una lingua franca (Rodríguez, 2012), su relevancia tiene que ver con la posibilidad de adquirir herramientas que faciliten el intercambio de bienes tangibles e intangibles con otros contextos culturales y sociales. Por tanto, las investigaciones dentro de las ciencias de la educación, se han enfocado en gran medida en explorar la importancia de mejorar y generar mejores formas de su enseñanza (Beltrán, 2017; Díaz, 2011; Medina, Melo y Palacios, 2013; Rueda y Wilburn, 2014). El presente ejercicio de investigación se orientó desde una de las aproximaciones teóricas y empíricas que han demostrado mejores resultados en la apropiación del inglés, esto es, el enfoque basado en tareas. Eso por esta razón que el objetivo de este trabajo es verificar el nivel de apropiación de la competencia discursiva entre los estudiantes de la Universidad de San Buenaventura Medellín del nivel nueve al diez del programa de inglés.

Específicamente, las tareas vivenciales han demostrado mayor impacto sobre la apropiación del componente discursivo del inglés, pues recurren al contexto del aprendiz, así como el uso de mejores prácticas didácticas que posibiliten que el aprendizaje sea significativo y se evidencie en cómo los estudiantes producen textos orales y escritos (Idiazabal y Larringan, 2004; Romero, González y Armijos, 2019). Dentro de los autores más citados y de mayor relevancia acerca del enfoque basado en tareas para el aprendizaje del inglés, se encuentra Nunan (2002). Los aportes de Nunan, vienen mejorándose en la medida que su teoría es 
usada cada vez más para delimitar modelos empíricos como el del presente ejercicio de investigación. En suma, la posición de Nunan fundamenta una visión ontológica, desde la que el aprendiz es un todo con su contexto y el sistema de enseñanza (Cantero, 2011; Díaz, 2010). De allí que las tareas y en especial la tarea comunicativa implique el "cómo" el estudiante que recibe la información y las aplique en las tareas orientadas por el facilitador, las cuales son pedagógicas y de la vida real (Nunan, 2002) se observen en la coherencia y ordenación de los textos orales y escritos del idioma inglés.

El problema de este trabajo surge bajo tres aspectos que tienen en común el mismo sentir, mejorar el aprendizaje del idioma inglés entre la comunidad estudiantil. El primer aspecto es presentado por las directivas de la Universidad quienes manifestaron una preocupación por los bajos resultados en las pruebas Saber Pro. El segundo aspecto, es analizado por los investigadores los cuales resaltaron la importancia de la apropiación de la competencia discursiva para desarrollar discursos orales y escritos según las situaciones inmediatas de los aprendices. El último aspecto, emerge después de hacer la revisión literaria respecto al enfoque basado por tareas para la enseñanza y aprendizaje del inglés como lengua extranjera, el cual sea trabajado con otras intenciones y metodologías como ya se mencionaron posteriormente.

\section{MARCO TEÓRICO}

Para la construcción de esta investigación se tomaron las categorías: Apropiación; Competencia discursiva; Tareas Vivenciales. A continuación, se describen estas categorías.

\section{LA APROPIACIÓN}

La apropiación del lenguaje se desarrolla a partir de la interacción con el otro (Montealegre y Forero, 2006). Esta se construye de forma oral y escrita bajo la abstracción de conceptos del contexto inmediato, dando lugar a la apropiación de palabras, las cuales se componen de significado y sonido (Vigotsky, 1993). El significado es una forma de recurrir al pensamiento para darle sentido a las palabras y el sonido es el habla externa de esas palabras (Duque y Packer, 2014).

Para Vigotsky el lenguaje juega un papel importante para la interacción social y el desarrollo humano de las personas (Mota y Villalobos, 2007). El lenguaje permite el intercambio de discursos activos para dar opiniones, debatir, solicitar algo, entre otras cosas. En este sentido, las funciones psicológicas superiores (atención, percepción, memoria, pensamiento, y lenguaje) son las que posibilitan el aprendizaje y la apropiación del lenguaje. Estas funciones se desarrollan de otras más básicas que compartimos con los animales, como lo es la imitación, los sentidos y las sensaciones (Orrú, 2012).

Para potencializar estas funciones dentro de la apropiación de la competencia discursiva en el aprendizaje del inglés se aborda la teoría de la Zona del Desarrollo Próximo formulada por Vigotsky. Esta teoría postula que el estudiante tiene un conocimiento previo y no es un ser vacío, y este conocimiento podría ser potencializado con la guía de otro actor para llegar a uno nuevo (Vigotsky 1979). Adicionalmente, también se establece como la distancia que hay entre el conocimiento real del aprendiz y aquel al que puede llenar gracias a la mediación de otro agente y sus herramientas, en este caso se propone que estas fuesen las tareas vivenciales y la interacción del docente para el desarrollo de la competencia discursiva.

\section{COMPETENCIA DISCURSIVA}

La comunicación es un proceso de interacción social donde se desarrolla un intercambio de discursos (Zaldua, 2006). Para tal proceso la competencia comunicativa hace parte de las competencias básicas comprendida como el 
conjunto de habilidades o capacidades que posibilitan una participación apropiada en situaciones comunicativas específicas (Hymes, 1972). Esto quiere decir, que la comunicación requiere de un discurso oral o escrito para expresar lo que se quiere decir, y de eso se encarga la competencia comunicativa.

La competencia discursiva desde el Marco Común Europeo de Referencia [MCER] (2001), hace referencia a: "la capacidad de ordenar el texto de manera secuencial y coherente" ( $p$. 24). De esta manera, la ordenación del discurso se comprende de: la ordenación coherente y la organización del texto. La ordenación coherente se da en función de los temas y perspectivas, oraciones ya establecidas, secuencia natural, relaciones de cause y efecto, control del discurso, la capacidad y la cantidad y la relación y el modo en que se presenta el texto. Por su lado, la organización del texto responde a cuestiones relativas a: cómo se estructura, cómo se cuenta, cómo se desarrolla y cómo se elabora la secuencia del texto.

\section{TAREAS VIVENCIALES}

Para comprender el concepto de tareas vivenciales se presenta lo que es el enfoque por tareas y posteriormente el concepto de vivencia para así llegar a la definición de este concepto.

Desde inicios del presente siglo surgió el enfoque basado en tareas para contribuir en la enseñanza y aprendizaje en el idioma inglés. Para Nunan, la tarea se refiere a actividades que potencialicen el uso real de la lengua según la situación inmediata (2013). Dentro de este enfoque, basado en tareas vivenciales, encontramos características y tipos que se describirán en el apartado de los resultados. Estas tareas generan ambientes de aprendizaje del idioma de una forma más auténtica y cotidiana entre los participantes. En este orden de ideas, para Cantero (2011), las tareas tienen un carácter contextual.
Voloshinov (1992) por su parte, entiende la vivencia como una conexión real con el conocimiento con una carga significativa de sentido, dicha conexión está enmarcada en el aquí, tiene, además, unas transformaciones internas en la persona en términos de existencia y de relación con su entorno. En otras palabras, la vivencia se compone de conocimiento, experiencia y contexto.

Las tareas vivenciales son así, tareas contextualizadas, enfocadas a resolver situaciones de contexto inmediato, que tienen en cuenta los conocimientos previos y las experiencias construidas por el estudiante. Desde esta visión, la tarea se lleva a cabo dentro de un ambiente de aprendizaje de forma contextualizada y de interés hacia el estudiante, de tal forma que esta se refleja y se resuelve en situaciones de contexto.

\section{METODOLOGÍA}

El presente estudio se abordó desde el paradigma sociocrítico desde un enfoque investigativo mixto con un alcance analítico. Según Alvarado y García (2008) "el paradigma sociocrítico construye una crítica social con un fuerte carácter autorreflexivo" (p. 190). Los autores afirmaron que este modelo se orienta a resolver problemáticas de conjuntos específico de personas y con ello desea alcanzar independencia racional y libertad. Así, este paradigma promueve la transformación social entre las comunidades que buscan cambios.

Dentro de un enfoque mixto se encuentran cinco propósitos inmersos para su desarrollo: "triangulación, complementariedad, iniciación, desarrollo y expansión" (Guelmes y Nieto, 2015, p. 25). Todo como rigurosidad del enfoque, ya que se parte de los dos enfoques, cualitativa y cuantitativa. Se debe considerar, además, que el trabajo investigativo se desarrolló bajo la investigación-acción, la cual es descripta por Kurl Lewin (citado por Vidal y Rivera, 2007, p. 1). Además, en la misma línea Muñoz, 
Quintero y Munévar (2002) proponen acerca de la investigación mixta desde el modelo del interaccionismo simbólico:

Como una forma de indagación introspectiva colectiva emprendida por participantes en situaciones sociales con objeto de mejorar la racionalidad y la justicia de sus prácticas sociales o educativas, así como su comprensión de esas prácticas y de las situaciones en que éstas tienen lugar (párr. 13)

La población seleccionada para este trabajo estuvo conformada por estudiantes de la Universidad de San Buenaventura Medellín que estuvieran cursando los niveles de inglés y la muestra que se tomó fue el grupo de estudiantes matriculados en el nivel noveno y que culminaron el nivel décimo, así la muestra estuvo conformada por ocho participantes. Se tomó esta muestra, ya que muchos estudiantes de pregrado y posgrado toman una prueba de clasificación quedando ubicados en niveles sexto, séptimo y octavo.

Con el fin de dar respuesta a la pregunta de esta investigación, las técnicas e instrumentos de recolección de información que fueron utilizados consistieron en: un pretest y un postest aplicados al inicio y final de cada nivel. Para la información cualitativa se tomó en cuenta un diario de campo, los planes de curso, la entrevista y un grupo focal entre los participantes con preguntas formuladas por los investigadores. En el diario de campo se consignó la información recopilada durante el desarrollo de las clases. A continuación, se detallan las técnicas e instrumentos de registro en relación con los objetivos específicos de la investigación

Tabla 1. Técnicas e instrumentos de recolección

\begin{tabular}{lll}
\hline \multicolumn{1}{c}{ Objetivo especifico } & \multicolumn{1}{c}{ Técnica } & \multicolumn{1}{c}{ Instrumento de registro } \\
\hline $\begin{array}{l}\text { Identificar las dificultades y fortalezas } \\
\text { de los estudiantes para la apropiación } \\
\text { de la competencia discursiva. }\end{array}$ & Test 1,2,3 y 4. & Papel y lápiz (pre-test y pos-test). \\
\hline $\begin{array}{l}\text { Caracterizar las tareas vivenciales } \\
\text { propuestas por el docente dentro del } \\
\text { desarrollo de las clases. }\end{array}$ & Revisión documental. & Plan de curso. \\
\hline $\begin{array}{l}\text { Valorar las tareas vivenciales } \\
\text { propuestas por el docente en relación } \\
\begin{array}{l}\text { a la apropiación de la competencia } \\
\text { discursiva. }\end{array}\end{array}$ & Entrevista. & Diario de campo. \\
\hline
\end{tabular}

Finalmente, para analizar e interpretar la información recolectada se usó la técnica de triangulación, con el fin de buscar convergencias y corroborar los resultados mediante el método y alcance de la investigación-acción.

\section{RESULTADOS Y DISCUSIÓN}

Los resultados se discuten a la luz de los objetivos específicos y, finalmente, se presenta el alcance de este trabajo, es decir verificar el nivel de apropiación de la competencia discursiva entre los estudiantes de la Universidad de San Buenaventura Medellín del noveno al décimo nivel de inglés a través de tareas vivenciales.

\section{DIFICULTADES Y FORTALEZAS DE LAS ESTUDIANTES PARA LA APROPIACIÓN DE LA COMPETENCIA DISCURSIVA}

Las tareas vivenciales orales y escritas propuestas en clase dentro de los niveles 
noveno y décimo correspondían a las temáticas de cada uno de los niveles. Para la estudiante 4"Ios temas y tareas del nivel décimo fueron más complejos que los del nivel noveno". Mientras que la participante 8 "los contenidos y tareas eran muy contextualizados acorde al nivel en que estábamos cursando". Con estas respuestas por parte de las participantes se analizó los diferentes instrumentos evidenciando avances en el nivel de apropiación de la competencia discursiva como se mostrará más adelante. Es decir que, se requiere de niveles de complejidad según el nivel de apropiación al que se quiere llegar.

Para Nunan (2013), la dificultad de la tarea se debe centrar en tres factores: estudiante, tarea e insumos. En relación al aprendiz, se tuvo presente sus conocimientos previos, se motivó a resolver la tarea, pero también él aprendió a su ritmo y al requerido por el docente. Para la tarea se partió de un objetivo claro entre los participantes, se presentó algunos pasos en su desarrollo y estaba contextualizada dando lugar a una mejor apropiación entre los aprendices. Los insumos se presentaron de forma clara, se dieron pistas contextualizas y fueron familiares para los estudiantes dentro del contenido diario de cada clase. El nivel de complejidad de las tareas vivenciales se evidenció en el objetivo de cada tarea. Un ejemplo tomado del plan de curso del nivel noveno es "describir mi niñez y adolescencia" esto implicó a que los participantes hicieran uso del pasado simple, además de encontrar el vocabulario adecuado para expresar lo que querían decir sobre los juegos y bromas. Otro ejemplo del nivel de complejidad para el curso décimo "presentar una posible solución a un problema global", esta tarea implicó para los estudiantes hacer uso del pasado, presente y pasado perfecto, condicionales, tiempos futuros y vocabulario en relación al problema seleccionado para presentar.
En la solución de las tareas orales y escritas se observó que los participantes tenían presentaban dificultades al escribir o pronunciar algunos verbos en tiempo pasado, además incurrían error al usar las preposiciones y signos de puntuación. Esta situación mostró avances al monitorear los efectos de las tareas vivenciales tal como se pudo constatar en las entradas del diario de campo. Es de resaltar que todos los participantes comprendían las instrucciones de las tareas y la llegaban a resolver por completo a pesar de presentar errores de escritura 0 pronunciación.

\section{CARACTERÍSTICAS DE LAS TAREAS VIVENCIALES}

En este estudio las tareas vivenciales presentaron cuatro características específicas que la diferencia de otro tipo de tarea: enganche, objetivo comunicativo, integración de habilidades y significativa y focalizada. El enganche es una categoría emergente que apareció en algunos instrumentos aplicados para esta investigación. Los participantes nombraron como enganche a los actividades o juegos que se presentaban al inicio de cada sesión de clase. Estas actividades o juegos eran utilizados por parte de los investigadores para activar los conocimientos previos de los estudiantes, adicionalmente para introducir la temática o el vocabulario de la clase.

Otra característica fue que el objetivo de la tarea era comunicativo (Modéer, 2010). Dentro de cada clase había un objetivo comunicativo que determinó la complejidad y el tema de la tarea. Dado que la competencia discursiva es la capacidad de producir discursos orales y escritos con coherencia los objetivos de las tareas vivenciales buscaban comunicar de forma oral o escrita una situación de contexto (Pulido y Muñoz, 2011). También se encontró como característica la integración de habilidades. Según Nunan (2013) la tarea integra componentes pragmáticos, sociolingüísticos y lingüísticos. Para resolver la tarea vivencial los 
participantes tuvieron los insumos de actividades de lectura y escucha en relación a la tarea. Esta integración de habilidades surgió como una segunda categoría emergente al presentarse en algunos de los instrumentos que se aplicaron. Esta característica permitió la solución de las tareas entre los estudiantes de una manera más cómoda y segura.

Como última característica se encontró que las tareas vivenciales eran significativas $y$ focalizadas para los aprendices. Ausubel (1976), estipuló que el aprendizaje significativo es la constitución de un nuevo conocimiento partiendo de conceptos previos con la ayuda del entorno. La tarea fue significativa para los participantes, ya que se tomaron sus conocimientos previos y gracias a la mediación de los investigadores (aplicación de tareas vivenciales y el contexto inmediato), de esta forma hubo una apropiación de la competencia discursivo. En esta misma línea Vygotsky (1993), manifestó que el lenguaje guía las acciones. En tal sentido, las tareas ya mencionadas fueron el lenguaje de mediación entre los aprendices y los docentes para llegar a una construcción de nuevos conocimientos que se dio en la solución de las tareas.

\section{VALORACIÓN DE LAS TAREAS VIVENCIALES}

Ante la valoración de las tareas vivenciales la estudiante 4 dijo "las tareas del nivel décimo presentaron más dificultad comparadas con las del nivel noveno", esto significó que hay niveles de dificultad según la apropiación a la que se desea llegar. Para los participantes 5 y 6 "los temas de las tareas fueron esenciales e interesantes que permiten tener una conversación en el diario vivir". Nunan (2013), mencionó que toda tarea debe ser de interés para los aprendices. Los anteriores participantes vieron las tareas interesantes, debido a que se abordaron temas cotidianos. Esto posibilitó a que comprendieron mejor las tareas y pudieran resolverla.
Al estudiar los textos del diario de campo y del grupo focal para valorar las tareas emergieron las categorías confianza y seguridad. Los participantes expresaron que "las tareas vivenciales contribuyeron a que nos sintiéramos con confianza y seguridad para comunicar nuestras ideas en el idioma ingles" Según MacClelland (2014), "la autoconfianza es la creencia o punto de vista que cada persona tiene de sí mismo" (p. 6). En este sentido, las tareas vivenciales como mediación para la apropiación de la competencia discursivo fue un apoyo para que los estudiantes tuvieran confianza y seguridad para expresarse de manera oral y escrita.

\section{NIVEL DE APROPIACIÓN DE LA COMPETENCIA DISCURSIVA CON LA APLICACIÓN DE LAS TAREAS VIVENCIALES}

En consideración a que el objetivo de este trabajo de investigación fue verificar el nivel de apropiación de la competencia discursiva con la aplicación de las tareas vivenciales entre los estudiantes de la Universidad de San Buenaventura Medellín del nivel noveno al décimo en el programa de inglés, para garantizar la validez de este objetivo, primero se identificó las dificultadas y fortalezas de los participantes en relación con la competencia discursiva, luego se caracterizaron las tareas vivenciales presentadas por los investigadores $\mathrm{y}$, finalmente, se procedió a valorarlas. Ahora bien, para verificar el nivel de apropiación se aplicaron un pre-test y un pos-test al inicio y final de cada nivel.

Estos test estaban diseñados para una tarea escrita y oral sobre temas específicos para cada nivel. Para empezar, se aplicó el pretest el primer día de cada curso. Luego, como mediación se implementaron las tareas vivenciales. Y, al final, se aplicó el pos-test. La escala utilizada para esto fue de 0 a 50 puntos usándose rúbricas analíticas. A continuación, se discrimina el nivel de apropiación de la competencia discursiva y algunas evidencias. 
Tabla 2. Resultados obtenidos por los estudiantes del nivel 9 del programa de inglés durante el desarrollo de la investigación.

\begin{tabular}{ccccccc}
\hline Participante & Tarea oral & $\begin{array}{c}\text { Tarea } \\
\text { escrita }\end{array}$ & $\begin{array}{c}\text { Total Pre- } \\
\text { test }\end{array}$ & Tarea oral & $\begin{array}{c}\text { Tarea } \\
\text { escrita }\end{array}$ & $\begin{array}{c}\text { Total Pos- } \\
\text { test }\end{array}$ \\
\hline 1 & 20 & 23 & $\mathbf{4 3}$ & 22 & 24 & $\mathbf{4 6}$ \\
2 & 8 & 13 & $\mathbf{2 1}$ & 22 & 19 & $\mathbf{4 1}$ \\
3 & 19 & 12 & $\mathbf{3 1}$ & 22 & 20 & $\mathbf{4 2}$ \\
4 & 16 & 22 & $\mathbf{3 8}$ & 23 & 24 & $\mathbf{4 7}$ \\
5 & 11 & 14 & $\mathbf{2 5}$ & 21 & 20 & $\mathbf{4 1}$ \\
6 & 19 & 15 & $\mathbf{3 4}$ & 23 & 22 & $\mathbf{4 5}$ \\
7 & 14 & 20 & $\mathbf{3 4}$ & 23 & 24 & $\mathbf{4 7}$ \\
8 & 20 & 20 & $\mathbf{4 0}$ & 25 & 24 & $\mathbf{4 9}$ \\
\hline
\end{tabular}

Tal como se evidencia en la tabla 2, todos los participantes en este estudio avanzaron. La participante 1 avanzó tres puntos, por su parte la participante 2 obtuvo 20 puntos. Esto sugiere que el nivel de apropiación entre los estudiantes no es el mismo y los tres factores anteriormente descritos (estudiante, tarea e insumos) influyeron en el nivel de apropiación.

Al revisar los resultados obtenidos en el nivel 10 se aprecia lo siguiente.

Tabla 3. Verificación nivel de apropiación nivel 10 del programa de inglés

\begin{tabular}{ccccccc}
\hline Participante & Tarea oral & $\begin{array}{c}\text { Tarea } \\
\text { escrita }\end{array}$ & $\begin{array}{c}\text { Total Pre- } \\
\text { test }\end{array}$ & Tarea oral & $\begin{array}{c}\text { Tarea } \\
\text { escrita }\end{array}$ & $\begin{array}{c}\text { Total Pos- } \\
\text { test }\end{array}$ \\
\hline 1 & 19 & 20 & $\mathbf{3 9}$ & 19 & 20 & $\mathbf{3 9}$ \\
2 & 14 & 15 & $\mathbf{2 9}$ & 18 & 20 & $\mathbf{3 8}$ \\
3 & 17 & 15 & $\mathbf{3 2}$ & 18 & 18 & $\mathbf{3 6}$ \\
4 & 17 & 18 & $\mathbf{3 5}$ & 20 & 23 & $\mathbf{4 3}$ \\
5 & 10 & 14 & $\mathbf{2 4}$ & 13 & 18 & $\mathbf{3 1}$ \\
6 & 20 & 20 & $\mathbf{4 0}$ & 22 & 20 & $\mathbf{4 2}$ \\
7 & 14 & 15 & $\mathbf{2 9}$ & 14 & 18 & $\mathbf{3 2}$ \\
8 & 21 & 19 & $\mathbf{4 0}$ & 25 & 25 & $\mathbf{5 0}$ \\
\hline
\end{tabular}

En esta ocasión la estudiante 1 no muestra avance, por su parte la estudiante 4 manifestó que gracias a la complejidad de las tareas obtuvo ocho puntos en su avance y 8 obtuvo diez puntos más respecto al total del pre-test. Las tareas vivenciales y temas para este nivel diez de inglés requerían manejo de todos los tiempos verbales y un amplio léxico sobre tecnología, quejas o reclamos, deseos y problemas sociales actuales. Esta situación indicó que el nivel de complejidad de las tareas vivenciales era más notorio para la estudiante 4 .
Al comparar las dos tablas se observó que los puntos de nivel de apropiación en el curso diez de inglés es menor al anterior, sin embargo, la mayoría de los participantes tuvieron algún avance en su nivel de apropiación de la competencia discursiva oral y escrita. Las siguientes figuras se presentan para ilustrar lo hasta aquí discutido. 
Figura 1. Pre-testescritura nivel 9 w

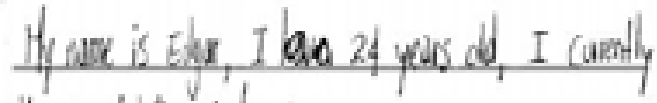

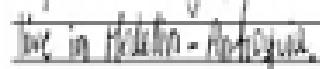

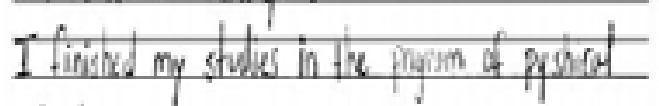

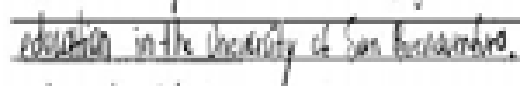

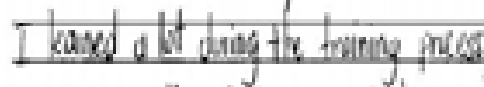

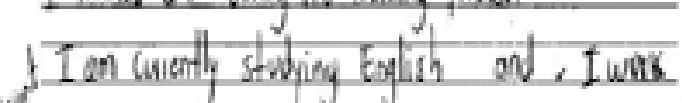

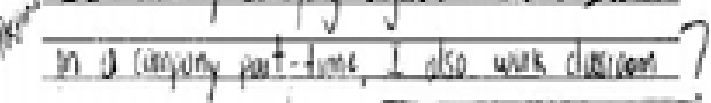
spoat in schools

Figura 2. Pos-test escritura nivel 9

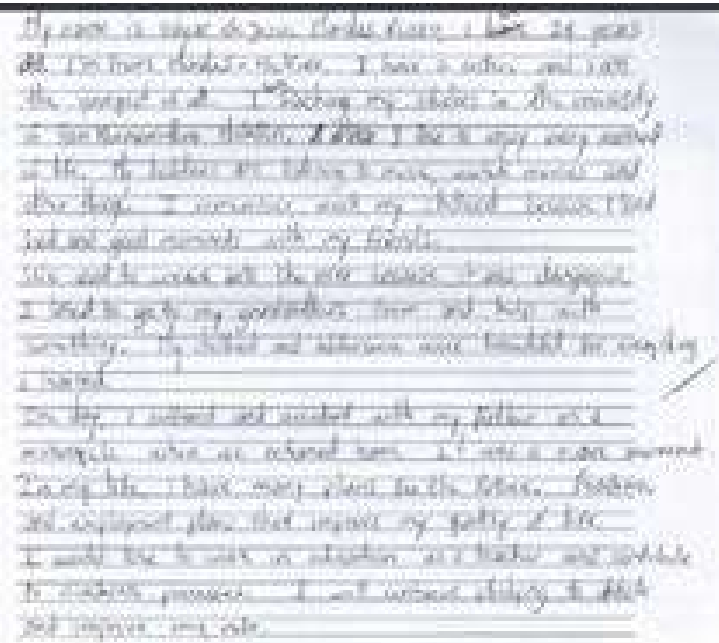

Figura 3. Pre-test escritura nivel 10

\section{The fups:}

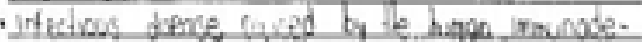

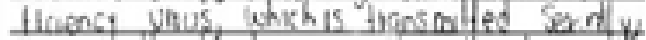

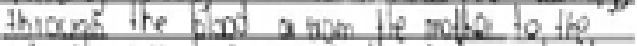

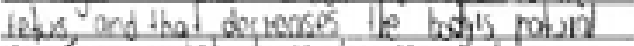
detenses antil If henches to candete difopporiners

The ereegences of this ins ap ditgrise

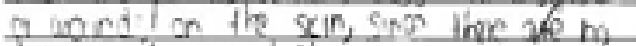
detemsos to peifh ith

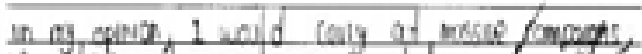

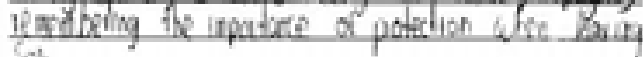

Figura 4. Pos-test escritura nivel 10

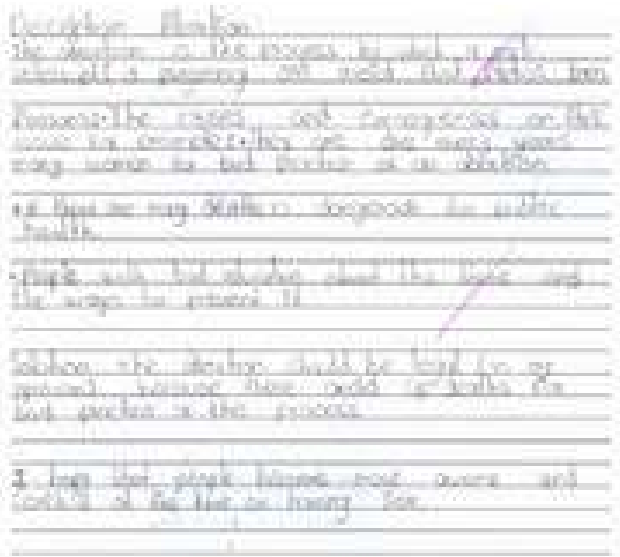

Figura 5. Rúbrica Pretest oral nivel 9

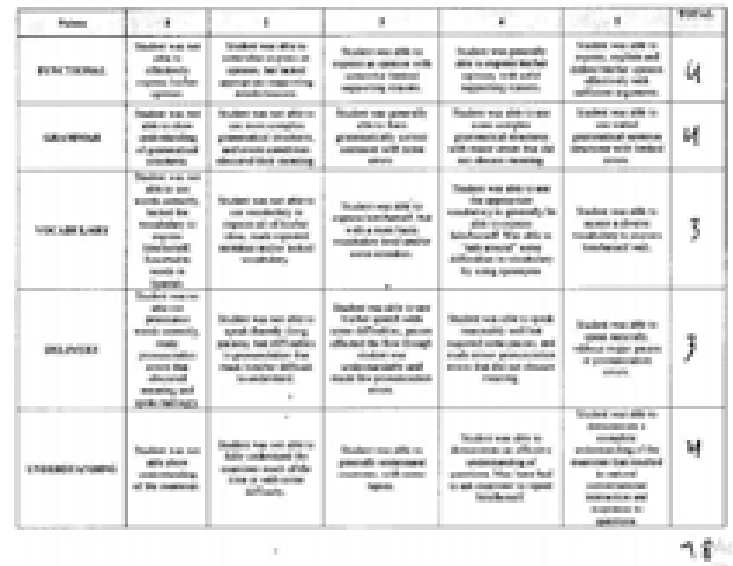

Figura 6. Rúbrica Pos-test oral nivel 9

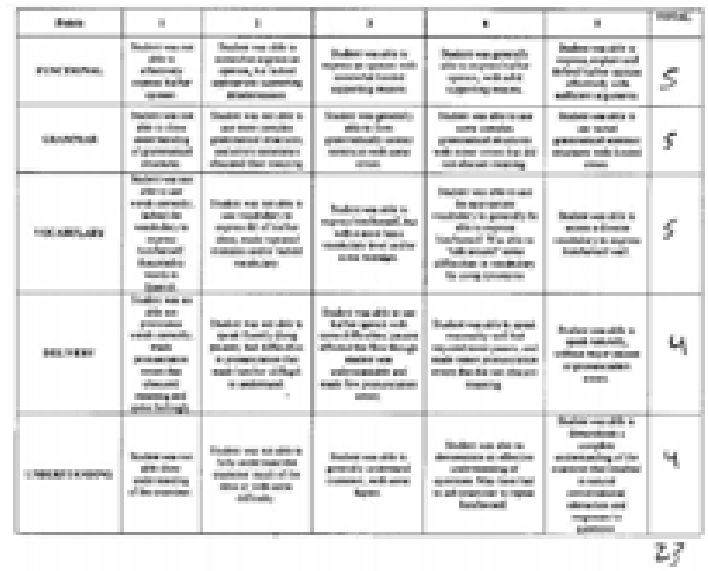

Tal como puede apreciarse, en la figura 1 el participante escribió ideas cortas, sobre su pasado y presente, con baja coherencia reflejando falencias en el orden y selección de las palabras. La figura 2 presenta la biografía del mismo participante y en esta ocasión se aprecia 
un avance notable tanto en la coherencia y fluidez en las ideas como en la extensión. Las figuras 3 y 4 muestran los resultados de otro de los participantes, en la figura 3 este describió un problema social definiendo el problema usando apreciaciones personales. El pos-test de la figura 4 mostró una definición más clara y soportada por razones, además planteó una posible solución a esta problemática. Las figuras 5 y 6 corresponden al pre y pos-test oral del nivel noveno en donde es posible apreciar un avance de cinco puntos en el resultado final de la prueba. Los anteriores hallazgos evidencian que la mediación mediante tareas vivenciales contribuye al desarrollo de la competencia discursiva.

\section{CONCLUSIONES}

El aprendizaje del idioma inglés ha tomado tanta relevancia que el Ministerio de Educación publicó en el año 2015 "los derechos básicos de aprendizaje para la enseñanza del inglés". Estos derechos son descritos desde primero de primaria hasta sexto grado de bachillerato. Alli se presentan las competencias que debe apropiar cada estudiante en su respectivo grado de escolaridad. Para la educación superior el Ministerio decretó en el 2016 la resolución 02041, donde se reglamenta los niveles de dominio de lengua extranjera para los programas de educación. De ahí, la preocupación de las directivas de la Universidad de San Buenaventura, Sede Medellín, de fortalecer el aprendizaje del inglés entre todos los estudiantes de la comunidad educativa. Por este motivo, se presenta la metodología didáctica de las tareas vivenciales para la apropiación del discurso oral y escrito, ya que se evidenció su efectividad con la población ya descrita al inicio de este artículo.

El enfoque por tareas en palabras de Cabrera (2017) especifica que: "brinda oportunidades a los aprendices de dominar el idioma de forma oral y escrita a través de actividades diseñadas para involucrar a los aprendices en el uso del idioma de una forma natural" (p. 10). Gracias a lo anterior surgió la importancia de realizar tareas vivenciales contextualizadas y del interés entre los aprendices las cuales promovieron la apropiación de la competencia discursiva entre ellos. Por lo tanto, contemplar el uso de tareas vivenciales puede contribuir en la apropiación de esta competencia de manera más singular y significativa. Para Ruiz y Pérez (2017), el aprendizaje se construye de forma vivencial donde se parte de experiencias previas y aptitudes entre los participantes que interactúan. En otras palabras, es en la vivencia (situación inmediata) donde en realidad se desarrolla el aprendizaje.

Por otro lado, este estudio encontró que las tareas vivenciales presentan niveles de complejidad según el nivel de apropiación al que se desea llegar y los conocimientos previos de los estudiantes. Adicionalmente, se halló que estas tareas presentaron unas características las cuales contribuyeron a su efectividad: objetivo comunicativo, enganche, integración de habilidades y significativas. Finalmente, esto permitió confianza para que los participantes comunicaran sus ideas $u$ opiniones de forma oral y escrito con coherencia, fluidez y orden.

\section{RECOMENDACIONES}

Dado los resultados arrojados en este estudio consideramos el discurso como una herramienta de comunicación la cual se presenta de manera oral y escrita. Para Rodríguez (2006) el discurso oral es "una práctica social que implica formas de acción entre las personas y que se articula a partir del uso lingüístico contextualiza-do" (p. 2). Por su parte Rico y Níkleva (2016), presentaron la escritura como un proceso lingüístico. Bajo estas dos perspectivas contemplamos que la apropiación de la competencia discursiva oral y escrita conversan entre sí y una se apoya de la otra, ya que la finalidad de ambas es comunicar algo, ya sea social, laboral o académico. 
Como segunda consideración presentamos la estructura que usamos en cada clase para que los participantes pudieran resolver la tarea vivencial. Primero, partimos de un objetivo claro que abarcara la tarea y los temas. Luego, presentábamos una actividad o juego para introducir el tema o el léxico que íbamos a trabajar, la finalidad de esto era activar los conocimientos previos de los participantes, ellos lo nombraron como el enganche. Posteriormente, venía una explicación del tema y vocabulario usando ejemplos cotidianos. Después de esto, los participantes desarrollaban actividades de escucha y lectura referente al tema. Esta integración de habilidades funcionó como insumo para resolver la tarea oral y escrita. Como parte final, se resolvía y socializaba la tarea vivencial.

Figura 7. Proceso de clase para aplicar las tareas vivenciales

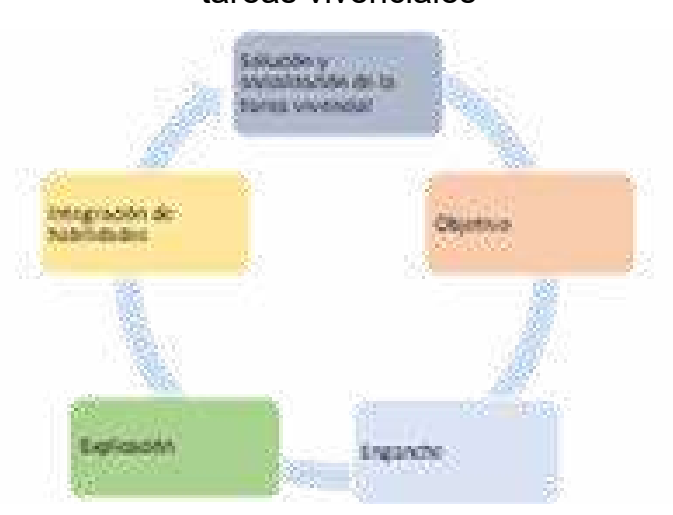

La última consideración que presenta este estudio empírico es la aplicación de las tareas vivenciales en todos los niveles de la educación donde se cuente con la enseñanza del idioma inglés. Dado que sería bueno validar la efectividad en otros contextos, además que las investigaciones realizadas en el enfoque basado en tareas concluyen en abordar este enfoque en diferentes contextos y perspectivas.

\section{REFERENCIAS}

Alvarado, L., y García, M. (2008). Características más relevantes del paradigma sociocrítico: su aplicación en investigaciones de educación ambiental y de enseñanza de las ciencias realizadas en el Doctorado de Educación del Instituto Pedagógico de Caracas. Sapiens, Vol. $9, \mathrm{~N}^{\circ} .2$, págs. 187-202.

Ausubel, D. (1976). Psicología educativa. Un punto de vista cognoscitivo. México: Trillas.

Beltrán, M. (2017). El aprendizaje del idioma inglés como lengua extranjera. Revista Boletín Redipe, Vol. 6-4, págs. 91-98

Cabrera, M. (2017). El Aprendizaje Basado en Tareas (ABT) en la Competencia Comunicativa de los / las estudiantes de los Cursos Súper Intensivos de la Universidad de las Fuerzas ArmadasESPE. (Tesis de maestría). Universidad central del Ecuador. Quito, Ecuador.

Cantero, V. (2011). La enseñanza de segundas lenguas a través de tareas: una propuesta didáctica para 1 de ESO bilingüe. Tendencias pedagógicas, Vol. 17, págs. 133.156 .

Díaz, M. (2010). Aprendizaje del inglés en adultos: una oportunidad de comunicación a través de la experiencia. (Tesis de grado). Pontificia Universidad Javeriana. Bogotá, Colombia.

Díaz, C. (2011). El aprendizaje del inglés y el uso de tecnologías: percepciones de estudiantes y profesores de inglés del nivel secundario chileno. Matices, Vol. 5, pp. 1-37.

Duque, M. P., y Packer, M. J. (2014). Pensamiento y lenguaje. El proyecto de Vygotsky para resolver la crisis de la Psicología. Tesis Psicológica, Vol. 9, №. 2, págs. 30-57.

Ministerio de Educación, Cultura y Deporte, Subdirección General de Cooperación Internacional. (2001). Marco común 
europeo de referencia para las lenguas: aprendizaje, enseñanza, evaluación. Madrid, España. Instituto Cervantes.

Flores, M. (2016). La globalización como fenómeno político, económico y social. Orbis, Vol. 12, №. 34, págs. 26-41.

Ministerio de Educación Nacional. (2015). Por el cual se reglamenta los Derechos básicos de aprendizaje para la enseñanza del inglés. Bogotá: Diario Oficial.

Ministerio de Educación Nacional. (3 de febrero, 2016). Resolución 02041. Por el cual se reglamenta los niveles de dominio para los futuros licenciados en relación al aprendizaje de una lengua extranjera. Bogotá: Diario oficial.

Guelmes, E. L., \& Nieto, L. E. (2015). Algunas reflexiones sobre el enfoque mixto de la investigación pedagógica en el contexto cubano. Revista Universidad y Sociedad, Vol. 7, №. 2, págs. 23-29.

Hymes, D. (1972). On communicative competence. In J. Pride \& J. Holmes (Eds.), Sociolinguistics (pp. 93-269). England: Penguin Books.

Idiazabal, I y Larringan, L. (2004). La competencia discursiva: noción clave para la didáctica de las lenguas del plurilingüismo. (tesis de maestría) Universidad del País Vasco. País Vasco, España.

MacLellan, E. (2014). How might Teachers enable Learner Self-confidence? A review study. Educational Review, Vol. $66, N^{0} .1$, págs. 59-74.

Medina, M., Melo, G., y Palacios, M. (2013). La importancia del aprendizaje del idioma inglés a temprana edad. Yachana, 2(2), pp. 191-195.

Modéer, T. (2010). Las actividades comunicativas en los textos didácticos. (Tesis de Maestría). Universidad de Dalarna. Dalarna, Suecia.

Montealegre, R., y Forero, L. (2006). Desarrollo de la lectoescritura: adquisición y dominio. Acta Colombiana de Psicología,
Vol. 9, №. 1, págs. 25-40.

Mota, C., \& Villalobos, J. (2007). El aspecto socio-cultural del pensamiento y del lenguaje: visión vygotskyana. Educere, Vol. 11, №. 38, págs. 411-418.

Muñoz, J., Quintero, J., y Munévar, R. (2002). Experiencias en investigación-acciónreflexión con educadores en proceso de formación en Colombia. Revista electrónica de investigación educativa, Vol. 4, No. 1.

Nunan, D. (2002). Diseño de tareas para una clase comunicativa. Cambridge; Press University.

Nunan, D (2013). Task-based language teaching. University of Hong Kong. Cambrige Press.

Orrú, S. (2012). Bases conceptuales del enfoque histórico-cultural para la comprensión del lenguaje. Estudios pedagógicos, Vol. 38, №. 2, págs. 337-353.

Pulido, R., y Muñoz, O. (2011). La competencia discursiva y el texto oral en lengua extranjera: un estudio de caso. Lengua y habla, Vol. 15, págs. 128-140.

Rico, A., y Níkleva, D. (2016). Análisis de la competencia lingüístico-discursiva escrita de los alumnos de nuevo ingreso del Grado de Maestro en Educación Primaria. Revista Signos, Vol. 49, №. 90, págs. 48-70. Doi: 10.4067/S071809342016000100003

Rodríguez, L. (2012). Establecimiento del Inglés como Lengua Franca. (Tesis de Maestría). Universidad de Almería. Almería, España.

Rodríguez, M. (2006). Consideraciones sobre el discurso oral en el aula. Enunciación, Vol. 11, №. 1, págs. 59-72. Doi: https://doi. org/10.14483/22486798.470

Romero, H., González, M., y Armijos, J. (2019). El estructuralismo en la enseñanza del inglés. Revista Boletín Redipe, Vol. 8, $\mathrm{N}^{\circ}$. 8, págs. 101-106.

Rueda, M., y Wilburn, M. (2014). Enfoques 
teóricos para la adquisición de una segunda lengua desde el horizonte de la práctica educativa. Perfiles educativos, Vol. 36, № 143.

Ruiz, D., \& Pérez, J. (2017). Aprendizaje experiencial, una herramienta estratégica en el desarrollo de las competencias organizacionales. (tesis de maestría). Universidad Militar de Nueva Granada. Bogotá, Colombia.

Vidal, L., \& Rivera, N. (2007). Investigaciónacción. Educación Médica Superior, Vo. 21, №. 4, págs. 1-15.

Voloshinov, V. (1992). El Marxismo y la filosofía del lenguaje. Los principales problemas de método sociológico en la ciencia del lenguaje. Madrid: Alianza editorial S.A.

Vygotsky, L. (1979). El desarrollo de los procesos psicológicos superiores. Estados Unidos: Harvard Press.

Vygotsky, L. (1993). Pensamiento y lenguaje. Madrid: Visor.

Zaldua, A. (2006). El análisis del discurso en la organización y representación de la información-conocimiento: elementos teóricos. Acimed, Vol. 14, №. 3, págs. 1-16. 